\title{
Asymmetric Morita-Baylis-Hillman Reaction Catalyzed By Isophoronediamine-Derived Bis-(thio)urea Organocatalysts
}

\author{
Albrecht Berkessel,* Katrin Roland, Jörg M. Neudörfl \\ Institut für Organische Chemie der Universität zu Köln, Greinstrasse 4, D-50939 Köln, Germany.
}

\section{Supporting Information}

Materials. Commercial reagents were purchased from Sigma Aldrich, Fluka, Acros, Lancaster or NovaBioChem, and used as received. Solvents were distilled and dried prior to use following standard procedures. ${ }^{[1]}$ Air- and/or moisture-sensitive reactions were carried out in a mbraun Unilab glove box under argon atmosphere or in the fume hood using Schlenk technique. Chromatographic separations were performed using silica gel 60 (230-400 mesh) from MN GmbH \& Co.

Instrumentation. Nuclear magnetic resonance $\left({ }^{1} \mathrm{H}\right.$ and $\left.{ }^{13} \mathrm{C}\right)$ spectra were recorded on a Bruker AC250 (250 MHz), Bruker AC300 (300 MHz) or Bruker DPX300 (300 MHz) NMR spectrometer. FT-IR spectra were recorded on a Perkin-Elmer Paragon 1000. HPLC analyses were performed using Agilent 1100 Series or Merck Hitachi LaChrom HPLC instrument. GC analyses were performed using HP 6890 Series or HP $6890 \mathrm{~N}$ instrument and $\mathrm{N}_{2}$ as carrier gas. HRMS analyses were performed on a Finnigan MAT 900S spectrometer.

\section{(I) Catalysis experiments}

\section{General procedure for catalysis experiments}

$\operatorname{DABCO}(5.61 \mathrm{mg}, 50.0 \mu \mathrm{mol})$ and catalyst $1 \mathrm{~d}(35.6 \mathrm{mg}, 50.0 \mu \mathrm{mol})$ were dissolved in 2-cyclohexen-1one (3) $(96.5 \mu \mathrm{L}, 96.1 \mathrm{mg}, 1.00 \mathrm{mmol})$, and tridecane $(15.2 \mu \mathrm{L}, 11.5 \mathrm{mg}, 62.5 \mu \mathrm{mol})$ was added as internal standard. The mixture was cooled to $10^{\circ} \mathrm{C}$ on a coolable shaker plate and cyclohexanecarbaldehyde (2) $(30.1 \mu \mathrm{L}, 28.0 \mathrm{mg}, 250 \mu \mathrm{mol})$ was added. The mixture was shaken for $72 \mathrm{~h}$ at $10{ }^{\circ} \mathrm{C}$ and $400 \mathrm{rpm}$. Aliquots of $10.0 \mu \mathrm{L}$ were taken at intervals and analyzed by GC on chiral stationary phase. In case of HPLC analysis, the reaction was stopped after $72 \mathrm{~h}$ and the product isolated by column chromatography ( $n$-hexane : ethyl acetate $3: 1$ ). 
<smiles>O=C1CCCC=C1C(O)C1CCCCC1</smiles>

The racemic product was obtained following a literature procedure. ${ }^{[2]}$ GC (Varian CP-Chirasil-Dex, $\left.160{ }^{\circ} \mathrm{C}(33 \mathrm{~min})\right): \tau_{\mathrm{R}}=2.16 \mathrm{~min}$ (tridecane), $23.28 \mathrm{~min}((S)$-product, minor), $25.04 \mathrm{~min}((R)$-product, major). Absolute configuration was assigned by comparison of the retention times with those reported by Nagasawa et al. ${ }^{[3]}$

\section{2-(Hydroxy-phenyl-methyl)-cyclohex-2-en-1-one (Table 4, entry 2)}<smiles>O=C1CCCC=C1C(O)c1ccccc1</smiles>

The racemic product was prepared following a literature procedure. ${ }^{[2]}$ GC (Varian CP-Chirasil-Dex, $\left.160{ }^{\circ} \mathrm{C}(40 \mathrm{~min})\right): \tau_{\mathrm{R}}=2.16 \mathrm{~min}$ (tridecane), $30.13 \mathrm{~min}((S)$-product, minor), $31.96 \mathrm{~min}((R)$-product, major). Absolute configuration was assigned by comparison of the retention times with those reported by Nagasawa et al. ${ }^{[3]}$

$\underline{\text { 2-[Hydroxy-(4-nitro-phenyl)-methyl)]-cyclohex-2-en-1-one (Table 4, entry 3) }}$<smiles>O=C1CCCC=C1C(O)c1ccc([N+](=O)[O-])cc1</smiles>

The racemic product was prepared following a literature procedure. ${ }^{[4]} \operatorname{HPLC}(\operatorname{Merck}(R, R)$-Whelk O1, $n$ hexane/2-propanol 9:1, flow: $1.00 \mathrm{ml} / \mathrm{min}, 50 \mathrm{~min}): \tau_{\mathrm{R}}=27.66 \mathrm{~min}((S)$-product, minor), $37.15 \mathrm{~min}$ $((R)$-product, major). Absolute configuration was assigned by comparison of the retention times with those reported by Schaus et al. ${ }^{[5]}$ 
<smiles>COc1ccc(C(O)C2=CCCCC2=O)cc1</smiles>

The general procedure for the catalysis experiments was followed and the product isolated by column chromatography. The product was obtained as a highly viscous yellow liquid in $79 \%$ yield (46 mg, $198 \mu \mathrm{mol})$. Analytical data were in agreement with the literature. ${ }^{[6]} \operatorname{HPLC}(\operatorname{Merck}(R, R)$-Whelk O1, $n$ hexane/2-propanol 9:1, flow: $1.00 \mathrm{ml} / \mathrm{min}, 50 \mathrm{~min}$ ): $\tau_{\mathrm{R}}=29.41 \mathrm{~min}$ (minor), $33.12 \mathrm{~min}$ (major). Absolute configuration was not assigned.

\section{$\underline{\text { 2-(1-Hydroxy-2-methyl-propyl)-cyclohex-2-en-1-one (Table 4, entry 5) }}$}<smiles>CC(C)C(O)C1=CCCCC1=O</smiles>

The racemic product was prepared following a literature procedure. ${ }^{[4]}$ GC (Varian CP-Chirasil-Dex, $\left.130{ }^{\circ} \mathrm{C}(22 \mathrm{~min})\right): \tau_{\mathrm{R}}=4.91 \mathrm{~min}$ (tridecane), $17.95 \mathrm{~min}((S)$-product, minor), $19.52 \mathrm{~min}((R)$-product, major). Absolute configuration was assigned by comparison of the retention times with those reported by Nagasawa et al. ${ }^{[3]}$

\section{$\underline{\text { 2-(1-Hydroxy-3-phenyl-propyl)-cyclohex-2-en-1-one (Table 4, entry } 6 \text { ) }}$}<smiles>O=C1CCCC=C1C(O)CCc1ccccc1</smiles>

The general procedure for the catalysis experiments was followed and the product isolated by column chromatography. The product was obtained as a highly viscous yellow liquid in $52 \%$ yield (30 mg, $130 \mu \mathrm{mol}$ ). Analytical data were in agreement with the literature. ${ }^{[7]}$ HPLC (Daicel CHIRALCEL, OD-H, $n$-hexane/2-propanol $9: 1$, flow: $0.50 \mathrm{ml} / \mathrm{min}, 35 \mathrm{~min}): \tau_{\mathrm{R}}=18.76 \mathrm{~min}((S)$-product, minor), $37.15 \mathrm{~min}$ $((R)$-product, major). Absolute configuration was assigned by comparison of the retention times with those reported by Schaus et al. ${ }^{[5]}$ 
<smiles>O=C1CCC=C1C(O)c1ccccc1</smiles>

The racemic product was prepared following a literature procedure. ${ }^{[4]}$ GC (Varian CP-Chirasil-Dex, $\left.160{ }^{\circ} \mathrm{C}(29 \mathrm{~min})\right): \tau_{\mathrm{R}}=2.17 \mathrm{~min}$ (tridecane), $23.33 \mathrm{~min}, 25.20 \mathrm{~min}$. Absolute configuration was not assigned.

\section{2-(Cyclohexyl-hydroxy-methyl)-cyclopent-2-en-1-one (Table 4, entry 8)}<smiles>O=C1CCC=C1C(O)C1CCCCC1</smiles>

To a mixture of cyclohexanecarbaldehyde $(60.2 \mu \mathrm{L}, 500 \mu \mathrm{mol}, 1.00 \mathrm{eq})$, imidazole $(34.0 \mathrm{mg}, 500 \mu \mathrm{mol}$, 1.00 eq) in aqueous $\mathrm{NaHCO}_{3}$ solution $(1 \mathrm{M}, 2.00 \mathrm{~mL})$ and THF $(500 \mu \mathrm{L})$ was added 2-cyclopenten-1-one $(62.9 \mu \mathrm{L}, 750 \mu \mathrm{mol}, 1.50 \mathrm{eq})$, and the solution was stirred for $60 \mathrm{~h}$ at $\mathrm{rt}$. $\mathrm{HCl}(1 \mathrm{M}, 3.00 \mathrm{~mL})$ was added and the aqueous phase extracted with ethyl acetate $(3 \times 3.00 \mathrm{~mL})$. The organic phase was dried over $\mathrm{MgSO}_{4}$, filtered and the solvent was evaporated under reduced pressure. The racemic product was obtained as a highly viscous yellow liquid in $41 \%$ yield $(40.0 \mathrm{mg}, 206 \mu \mathrm{mol})$. Analytical data were in agreement with the literature. ${ }^{[8]} \mathrm{GC}$ (Varian CP-Chirasil-Dex, $160{ }^{\circ} \mathrm{C}(22 \mathrm{~min})$ ): $\tau_{\mathrm{R}}=2.17 \mathrm{~min}$ (tridecane), $17.53 \mathrm{~min}$ (minor), $18.59 \mathrm{~min}$ (major). Absolute configuration was not assigned.

\section{2-(Hydroxy-phenyl-methyl)-acrylic acid methyl ester (Table 4, entry 9)}<smiles>C=C(C(=O)OC)C(O)c1ccccc1</smiles>

The racemic product was prepared following a literature procedure. ${ }^{[2]}$ GC (Varian CP-Chirasil-Dex, $\left.135^{\circ} \mathrm{C}(35 \mathrm{~min})\right): \tau_{\mathrm{R}}=4.14 \mathrm{~min}$ (tridecane), $28.15 \mathrm{~min}, 29.50 \mathrm{~min}$. Absolute configuration was not assigned. 
<smiles>C=C(C(=O)OC)C(O)C1CCCCC1</smiles>

The racemic product was prepared following a literature procedure. ${ }^{[9]}$ GC (Varian CP-Chirasil-Dex, $\left.130{ }^{\circ} \mathrm{C} \quad(35 \mathrm{~min})\right): \tau_{\mathrm{R}}=4.91 \mathrm{~min}$ (tridecane), $25.97 \mathrm{~min}$ (minor), $26.83 \mathrm{~min}$ (major). Absolute configuration was not assigned.

\section{(II) Preparation of the bis-(thio)urea catalysts 1a, 1c, 1e and 1f.}

Preparation of $N$-Phenyl- $N^{\prime}-[(1 R, 3 S)-3-[[$ (phenylamino)oxomethyl]amino]methyl]-3,5,5-trimethylcyclohexyllurea (1a)

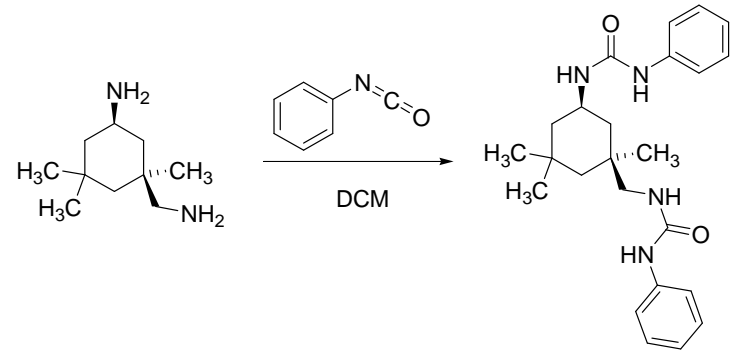

(1R,3S)-3-(Aminomethyl)-3,5,5-trimethyl-cyclohexylamine ( $860 \mu \mathrm{L}, 4.65 \mathrm{mmol}, 1.00 \mathrm{eq})$ was dissolved in dry DCM $(8.00 \mathrm{~mL})$, and phenylisocyanate $(1.02 \mathrm{~mL}, 9.34 \mathrm{mmol}, 2.01 \mathrm{eq})$ was added. A colorless precipitate formed immediately which was filtered off. It was recrystallized from $\mathrm{CHCl}_{3} / \mathrm{MeOH}$ to yield the desired bis-urea quantitatively $(1.90 \mathrm{~g}, 4.65 \mathrm{mmol})$ as colorless crystals suitable for $\mathrm{x}$-ray crystallography. $[\alpha]_{589}^{20}=-38.5^{\circ}(c=1.09, \mathrm{MeOH})$. mp 190-193 ${ }^{\circ} \mathrm{C} .{ }^{1} \mathrm{H}-\mathrm{NMR}\left(300 \mathrm{MHz}, \mathrm{DMSO}-\mathrm{d}_{6}\right): \delta=$ 0.82-1.11 (m; 12H), 1.12-1.25 (m; 1H), 1.52-1.70 (m; 2H), $2.87(\mathrm{dq} ; J=11.7 \mathrm{~Hz}, 5.85 \mathrm{~Hz}, 6.43 \mathrm{H}, 2 \mathrm{H})$, 3.71-3.89 (m; 1H), $5.96(\mathrm{~d} ; J=7.60 \mathrm{~Hz}, 1 \mathrm{H}), 6.21(\mathrm{t} ; J=5.85 \mathrm{~Hz}, 1 \mathrm{H}), 6.82-6.92(\mathrm{~m} ; 2 \mathrm{H}), 7.13-7.26$ $(\mathrm{m} ; 4 \mathrm{H}), 7.32-7.42(\mathrm{~m} ; 4 \mathrm{H}), 8.25(\mathrm{~s} ; 1 \mathrm{H}), 8.37(\mathrm{~s} ; 1 \mathrm{H}) .{ }^{13} \mathrm{C}-\mathrm{NMR}\left(75 \mathrm{MHz}, \mathrm{DMSO}-\mathrm{d}_{6}\right): \delta=23.2,27.5$, 31.5, 34.9, 36.0, 41.8, 42.3, 46.1, 46.8, 52.7, 117.3, 117.4, 120.8, 128.5, 128.6, 140.4, 140.5, 154.4, 155.3. FT-IR (CsI): $\widetilde{v}\left[\mathrm{~cm}^{-1}\right]=3334(\mathrm{~m}(\mathrm{br})), 2959(\mathrm{w}), 1646,1603,1560$ (all s), 1500, 1444, 1314, 1237 (all m), 758, 692 (both w). HRMS (ESI): Calcd. for $\left[\mathrm{C}_{24} \mathrm{H}_{32} \mathrm{~N}_{4} \mathrm{O}_{2} \mathrm{Na}\right]\left([\mathrm{M}+\mathrm{Na}]^{+}\right)$: 431.242, Found: $431.242( \pm 0.002 \mathrm{u})$. Anal. Calcd. for $\mathrm{C}_{24} \mathrm{H}_{32} \mathrm{~N}_{4} \mathrm{O}_{2}: \mathrm{C} 70.56, \mathrm{H} 7.90, \mathrm{~N} 13.71$ Found: C 70.54, H 8.04, N 13.71. 
Preparation of $N$-Phenyl- $N^{\prime}-[(1 R, 3 S)-3-[[[($ phenylamino $)$ thioxomethyl]amino]methyl]-3,5,5-trimethylcyclohexyl]thiourea (1c)

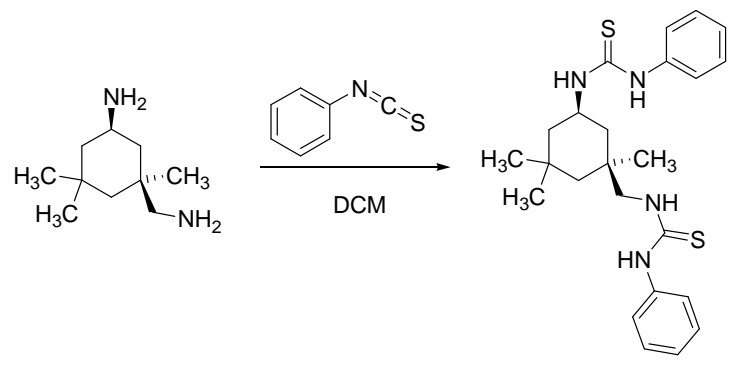

$(1 R, 3 S)$-3-(Aminomethyl)-3,5,5-trimethyl-cyclohexylamine (200 $\mu \mathrm{L}, 1.08 \mathrm{mmol}, 1.00 \mathrm{eq})$ was dissolved in dry DCM $(10.0 \mathrm{~mL})$, and phenylisothiocyanate $(262 \mu \mathrm{L}, 2.21 \mathrm{mmol}, 2.05 \mathrm{eq})$ was slowly added. The mixture was stirred for two hours at rt, the solvent removed under reduced pressure, and the resulting solid recrystallized from acetone. The desired bis-thiourea was obtained quantitatively (476 mg, $1.08 \mathrm{mmol})$ as colorless crystals suitable for x-ray crystallography. $[\alpha]_{589}^{20}=-33.2^{\circ}(c=0.69, \mathrm{MeOH}) . \mathrm{mp}$ 181-183 ${ }^{\circ} \mathrm{C} .{ }^{1} \mathrm{H}-\mathrm{NMR}\left(300 \mathrm{MHz}, \mathrm{CDCl}_{3}\right): \delta=0.78-1.13(\mathrm{~m} ; 12 \mathrm{H}), 1.14-1.24(\mathrm{~m} ; 1 \mathrm{H}), 1.77-2.01$ (m; 2H), $3.45(\mathrm{dq} ; J=5.55 \mathrm{~Hz}, 13.3 \mathrm{~Hz}, 29.4 \mathrm{~Hz}, 2 \mathrm{H}), 4.44-4.64(\mathrm{~m} ; 1 \mathrm{H}), 5.70$ (d; $J=7.97 \mathrm{~Hz}, 1 \mathrm{H}), 6.04(\mathrm{t}$; $\mathrm{J}=5.55 \mathrm{~Hz}, 1 \mathrm{H}), 7.02-7.48(\mathrm{~m} ; 10 \mathrm{H}), 7.91(\mathrm{~s} ; 1 \mathrm{H}), 8.01(\mathrm{~s} ; 1 \mathrm{H}) .{ }^{13} \mathrm{C}-\mathrm{NMR}\left(75 \mathrm{MHz}, \mathrm{CDCl}_{3}\right): \delta=23.5$, 27.7, 32.1, 35.0, 36.8, 41.2, 45.3, 47.8, 49.1, 59.1, 125.0, 125.4, 127.2, 127.5, 130.3, 130.4, 135.9, 136.0, 179.4, 181.2. FT-IR (ATR, film): $\tilde{v}\left[\mathrm{~cm}^{-1}\right]=3242$ (m(br)), 2951, 2951 (both m), 2088 (w(br)), 1705 (w), 1593 (m), 1528, 1490 (both s), 1446 (m), 1350, 1312, 1238, 1177 (all s), 907 (m), 729, 693 (both s). HRMS (ESI): Calcd. for $\left[\mathrm{C}_{24} \mathrm{H}_{32} \mathrm{~N}_{4} \mathrm{~S}_{2} \mathrm{Na}\right]\left([\mathrm{M}+\mathrm{Na}]^{+}\right)$: 463.197 , Found: 463.197 ( $\left.0.002 \mathrm{u}\right)$. Anal. Calcd. for $\mathrm{C}_{24} \mathrm{H}_{32} \mathrm{~N}_{4} \mathrm{~S}_{2}$ : C 65.41, H 7.32, N 12.71 Found: C 65.06, H 7.42, N 12.57.

Preparation of $N$-Cyclohexyl- $N^{\prime}-[(1 R, 3 S)-3-[[$ [cyclohexylamino)thioxomethyl]amino]methyl]-3,5,5$\underline{\text { trimethylcyclohexyl]thiourea }(\mathbf{1 e})}$

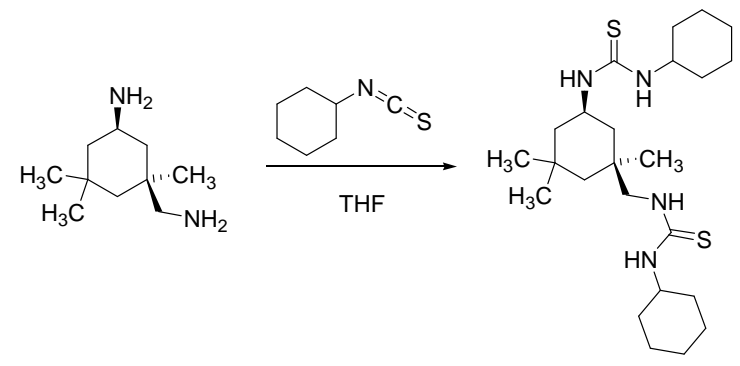


(1R,3S)-3-(Aminomethyl)-3,5,5-trimethyl-cyclohexylamine $\quad(1.00 \mathrm{~mL}, \quad 5.40 \mathrm{mmol}, \quad 1.00 \mathrm{eq}) \quad$ was dissolved in dry THF $(1.00 \mathrm{~mL})$, and cyclohexylisothiocyanate $(1.55 \mathrm{~mL}, 11.3 \mathrm{mmol}, 2.10 \mathrm{eq})$ was slowly added under argon atmosphere whereupon a yellow gel formed immediately. The solvent was removed under reduced pressure, and the resulting solid purified by column chromatography $\left(\mathrm{CHCl}_{3} / \mathrm{MeOH} 100: 5\right)$. The desired bis-thiourea was obtained in $65 \%$ yield $(1.60 \mathrm{~g}, 3.53 \mathrm{mmol})$ as a pale yellow solid. mp $139-141{ }^{\circ} \mathrm{C} .{ }^{1} \mathrm{H}-\mathrm{NMR}\left(300 \mathrm{MHz}, \mathrm{CDCl}_{3}\right): \delta=0.85-1.48(\mathrm{~m} ; 25 \mathrm{H}), 1.55-1.83(\mathrm{~m}$; $5 \mathrm{H}), 1.83-2.12(\mathrm{~m} ; 5 \mathrm{H}), 3.25(\mathrm{~s} ; 2 \mathrm{H}), 3.75-4.08(\mathrm{~m} ; 2 \mathrm{H}), 4.38-4.64(\mathrm{~m} ; 1 \mathrm{H}), 5.76-6.37(\mathrm{~m} ; 4 \mathrm{H}) .{ }^{13} \mathrm{C}-$ $\operatorname{NMR}\left(75 \mathrm{MHz}, \mathrm{CDCl}_{3}\right): \delta=24.2,24.7,24.8,25.4,27.8,32.1,32.9,33.0,33.2,35.0,36.5,41.3$, 45.7, 47.5, 48.0, 52.6, 57.7, 179.5. FT-IR (ATR, film): $\tilde{v}\left[\mathrm{~cm}^{-1}\right]=3253$ (w(br)), 2927 (m), 2851 (w), 2099 (w(br)), 1536 (s), 1448, 1359, 1255 (all m), 1062, 973, 889 (all w), 733 (s), 700 (m). HRMS (ESI): Calcd. for $\left[\mathrm{C}_{24} \mathrm{H}_{44} \mathrm{~N}_{4} \mathrm{~S}_{2} \mathrm{Na}\right]\left([\mathrm{M}+\mathrm{Na}]^{+}\right)$: 475.291, Found: $475.291( \pm 0.002 \mathrm{u})$.

Preparation of $N$-Tricyclo[3.3.1.1 $\left.1^{3,7}\right] \mathrm{dec}-1$-yl- $N^{\prime}-\left[\left\lceil(1 S, 5 R)-1,3,3\right.\right.$-trimethyl-5-[ऍ(tricyclo[3.3.1.1 $\left.{ }^{3,7}\right] \mathrm{dec}-1$ yl)aminolthioxomethyl]aminolcyclohexyl]methyl]thiourea (1f)

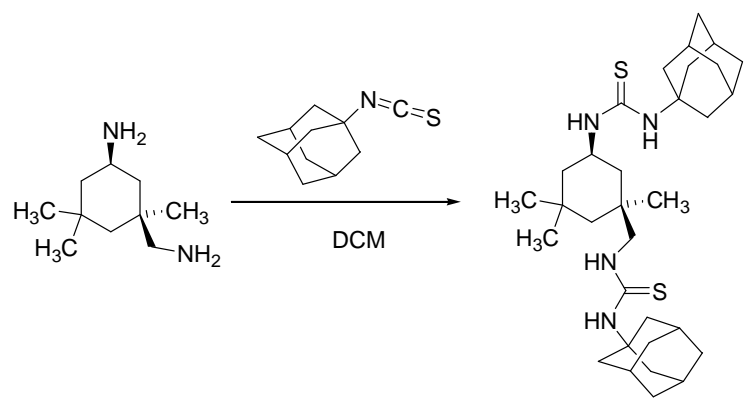

(1R,3S)-3-(Aminomethyl)-3,5,5-trimethyl-cyclohexylamine (100 $\mu \mathrm{L}, 540 \mu \mathrm{mol}, 1.00 \mathrm{eq})$ was dissolved in dry DCM (1.00 mL), and 1-adamantylisothiocyanate $(209 \mathrm{mg}, 1.08 \mathrm{mmol}, 2.00 \mathrm{eq})$ was slowly added under argon atmosphere. The mixture was stirred for two hours at rt, then the solvent removed under reduced pressure and the resulting solid purified by column chromatography $\left(\mathrm{CHCl}_{3} / \mathrm{MeOH} 100: 5\right)$. The desired bis-thiourea was obtained quantitatively (307 mg, $540 \mu \mathrm{mol})$ as a colorless solid. mp 180$182^{\circ} \mathrm{C} .{ }^{1} \mathrm{H}-\mathrm{NMR}\left(300 \mathrm{MHz}, \mathrm{CDCl}_{3}\right): \delta=0.92-1.33(\mathrm{~m} ; 12 \mathrm{H}), 1.58-1.79(\mathrm{~m} ; 12 \mathrm{H}), 1.92-2.07(\mathrm{~m} ; 13 \mathrm{H})$, 2.07-2.19 (m; 6H), $3.40(\mathrm{~s} ; 2 \mathrm{H}), 4.41-4.62(\mathrm{~m} ; 1 \mathrm{H}), 5.47(\mathrm{~d}(\mathrm{br}) ; 1 \mathrm{H}), 5.70-5.94(\mathrm{~m} ; 3 \mathrm{H}) .{ }^{13} \mathrm{C}-\mathrm{NMR}(75$ $\left.\mathrm{MHz}, \mathrm{CDCl}_{3}\right): \delta=23.9,27.8,29.4,32.1,35.0,36.1,36.5,41.8,42.5,45.8,47.9,49.1,54.0,179.9$. FTIR (ATR, film): $\tilde{v}\left[\mathrm{~cm}^{-1}\right]=3267$ (w(br)), 2907 (s), 2847 (m), 2208 (w(br)), 1529 (s), 1452 (m), 1355, 1340, 1300, 1234 (all s), 1101, 1075 (both m), 977 (w), 906, 727 (both s), 693, 643 (both m). HRMS (ESI): Calcd. for $\left[\mathrm{C}_{32} \mathrm{H}_{53} \mathrm{~N}_{4} \mathrm{~S}_{2}\right]\left([\mathrm{M}+\mathrm{H}]^{+}\right)$: 557.371, Found: $557.371( \pm 0.002 \mathrm{u})$. 
$\underline{\mathrm{X} \text {-ray crystallographic data for } 1 \mathrm{a} \text { and } 1 \mathrm{c}}$

\begin{tabular}{|l|c|c|}
\hline & 1a & 1c \\
\hline formula & $\mathrm{C}_{24} \mathrm{H}_{32} \mathrm{~N}_{4} \mathrm{O}_{2}$ & $\mathrm{C}_{24} \mathrm{H}_{32} \mathrm{~N}_{4} \mathrm{~S}_{2}$ \\
\hline$M_{\mathrm{r}}$ & 408.54 & 440.66 \\
\hline crystal dimensions $[\mathrm{mm}]$ & $0.3 \times 0.2 \times 0.2$ & $0.3 \times 0.1 \times 0.05$ \\
\hline crystal system & monoclinic & orthorhombic \\
\hline space group & $\mathrm{P} 2_{1}($ no. 4$)$ & $\mathrm{P}_{1} 2_{1} 2_{1}($ no. 19$)$ \\
\hline$a[\AA]$ & $9.244(7)$ & $7.5688(3)$ \\
\hline$b[\AA]$ & $9.5634(10)$ & $9.5287(4)$ \\
\hline$c[\AA]$ & $13.502(2)$ & $33.9726(14)$ \\
\hline$\alpha\left[{ }^{\circ}\right]$ & 90 & 90 \\
\hline$\beta\left[^{\circ}\right]$ & $106.642(6)$ & 90 \\
\hline$\gamma\left[{ }^{\circ}\right]$ & 90 & 90 \\
\hline$V\left[\AA^{3}\right]$ & $1143.6(2)$ & $2450.1(2)$ \\
\hline$\rho_{\text {calcd }}\left[\mathrm{g} \cdot \mathrm{cm}^{-3}{ }^{-3}\right]$ & 1.186 & 1.195 \\
\hline$Z$ & 4 & 4 \\
\hline radiation & Mo- $K_{\alpha}$ & Mo- $K_{\alpha}$ \\
\hline scan mode & $\varphi / \omega$ & $\varphi / \omega$ \\
\hline $2 \Theta_{\text {max }}\left[{ }^{\circ}\right]$ & 54 & 54 \\
\hline unique reflections & 2565 & 5183 \\
\hline observed reflections & 1812 & 3529 \\
\hline$R 1$ & 0.0803 & 0.040 \\
\hline$w R 2$ & 0.1063 & 0.071 \\
\hline$\rho_{\text {fin }}($ max $)\left[\mathrm{e} \cdot \AA^{-3}{ }^{-3}\right]$ & 0.242 & 0.248 \\
\hline $\mathrm{CCDC}$ depository no. & 605967 & 605968 \\
\hline & & \\
\hline
\end{tabular}




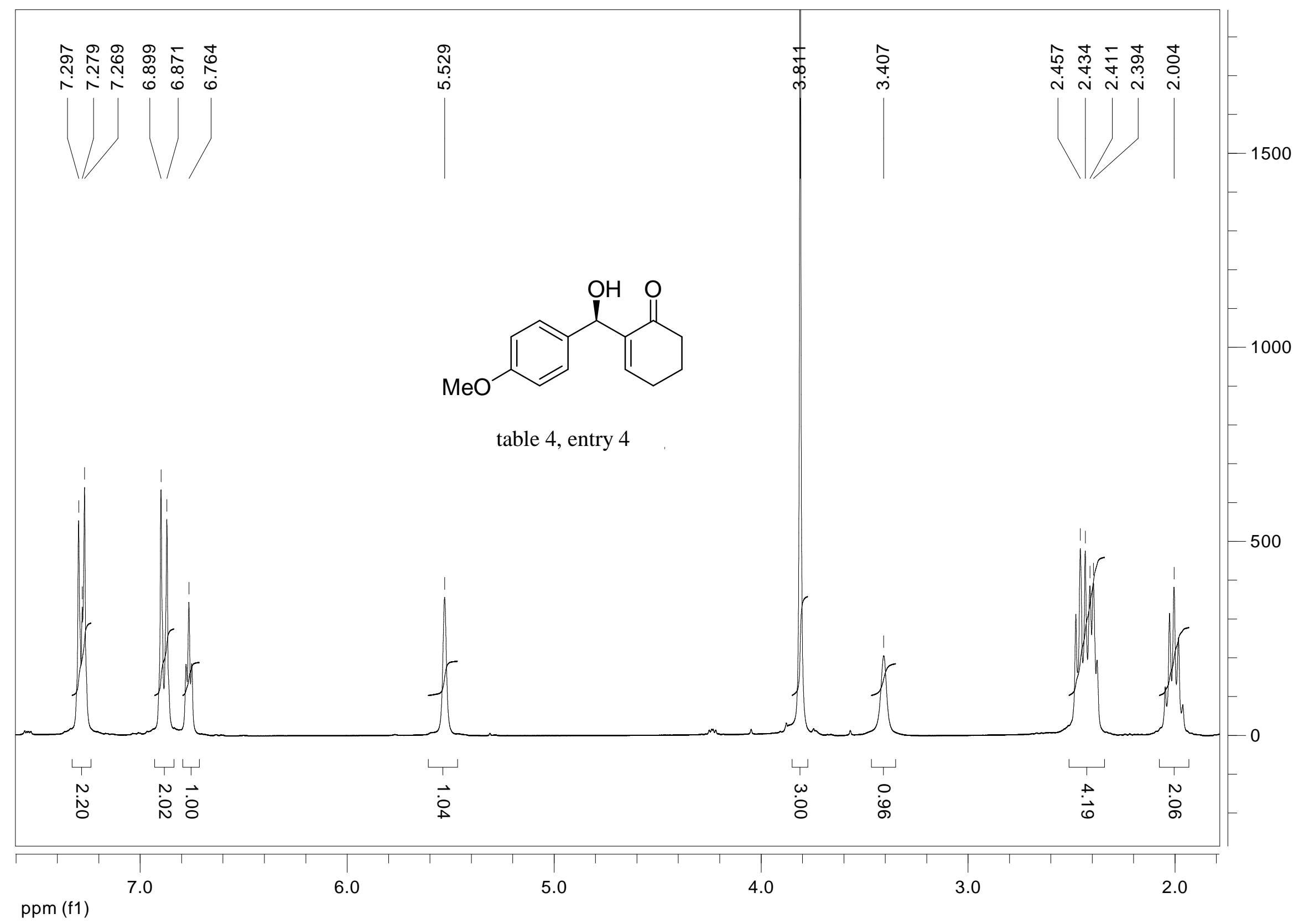




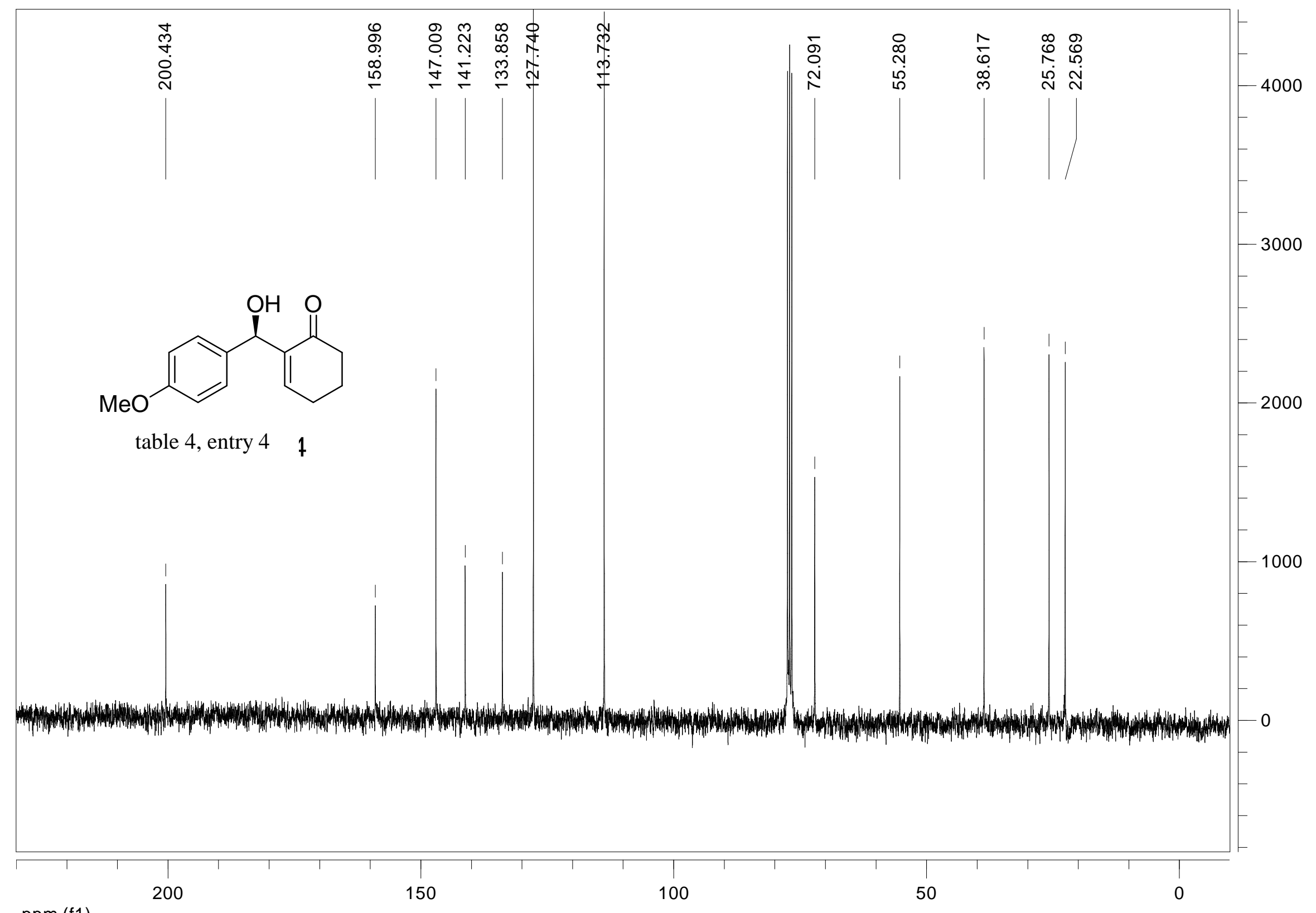

ppm (f1) 


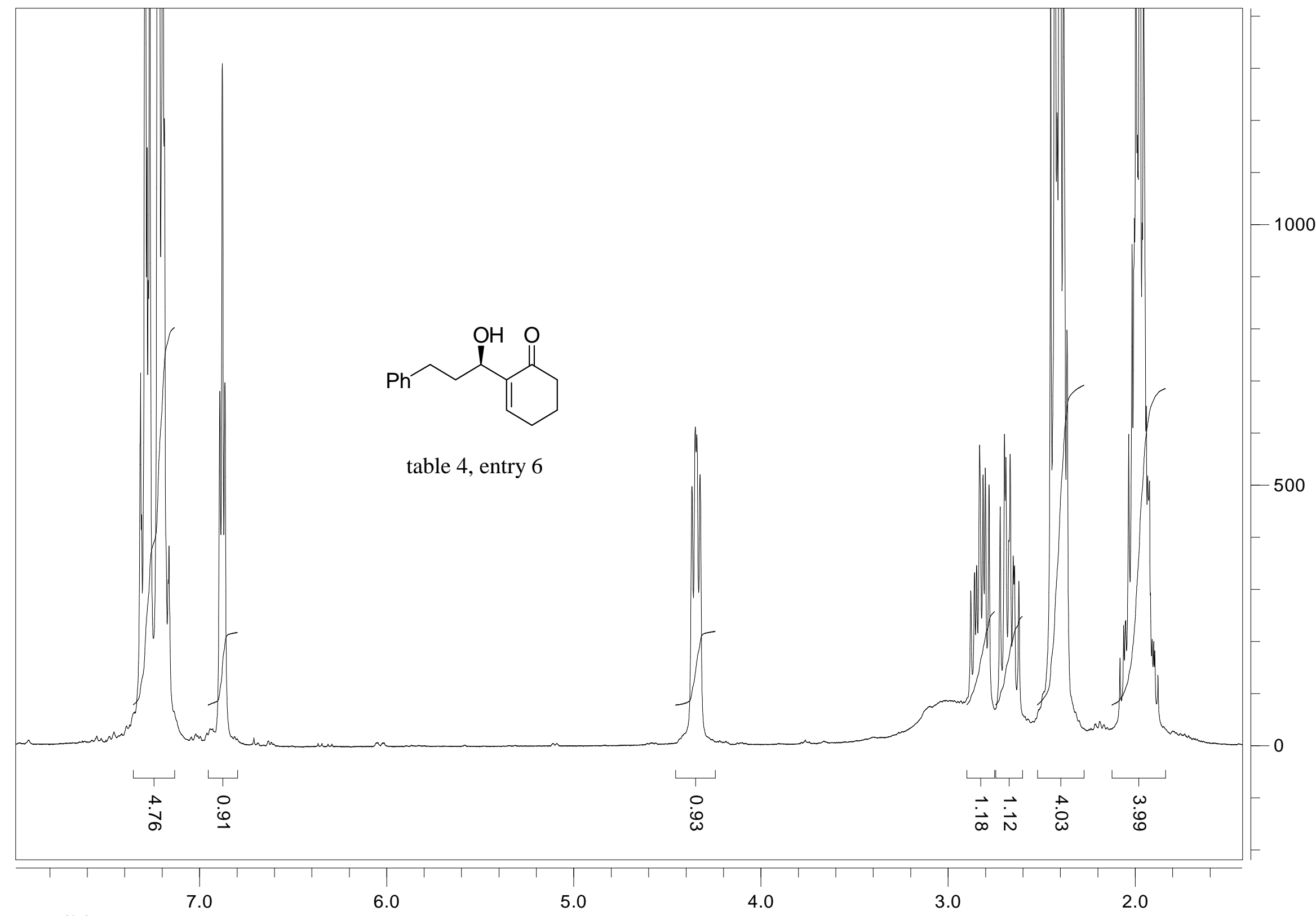

ppm (f1) 


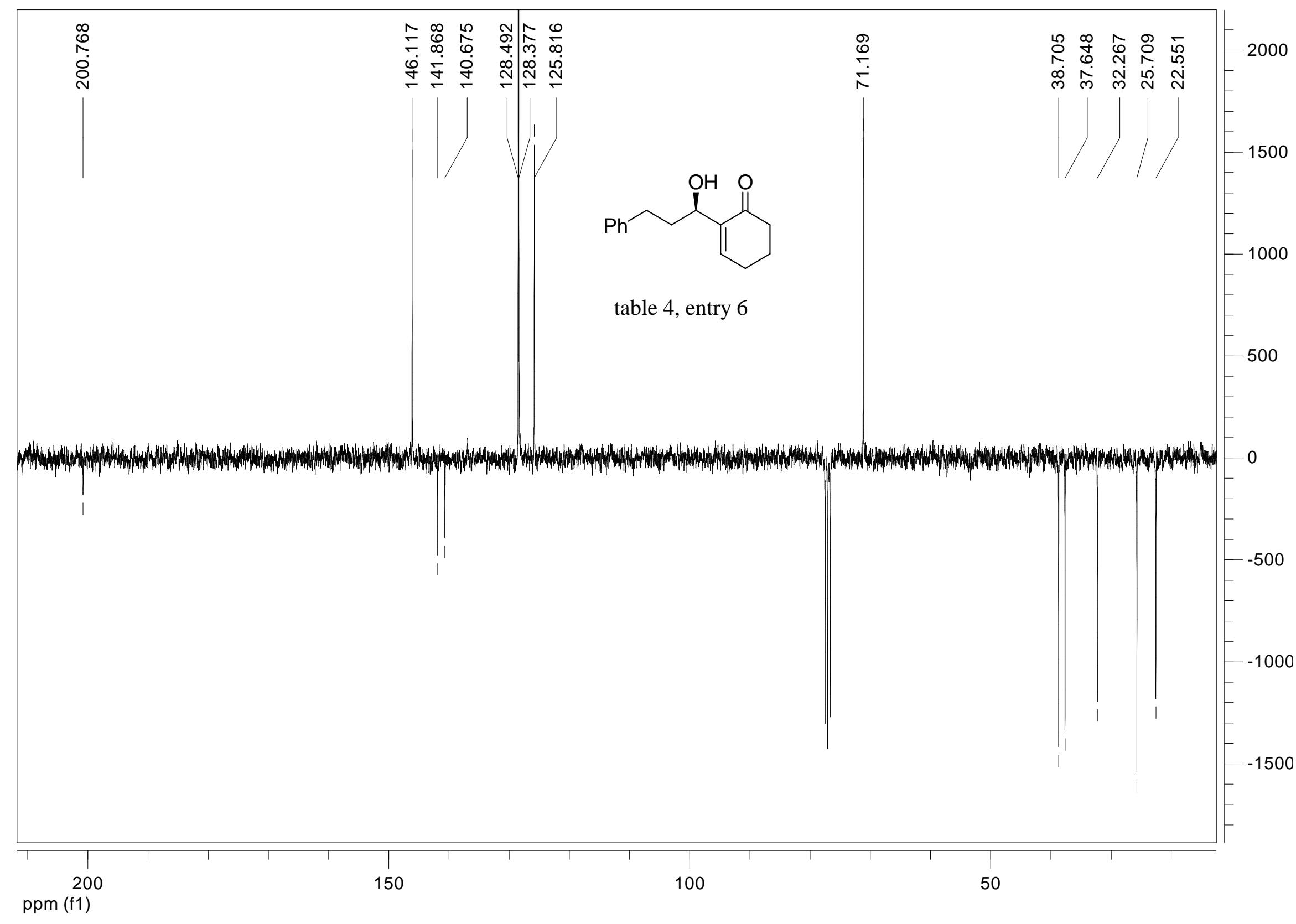




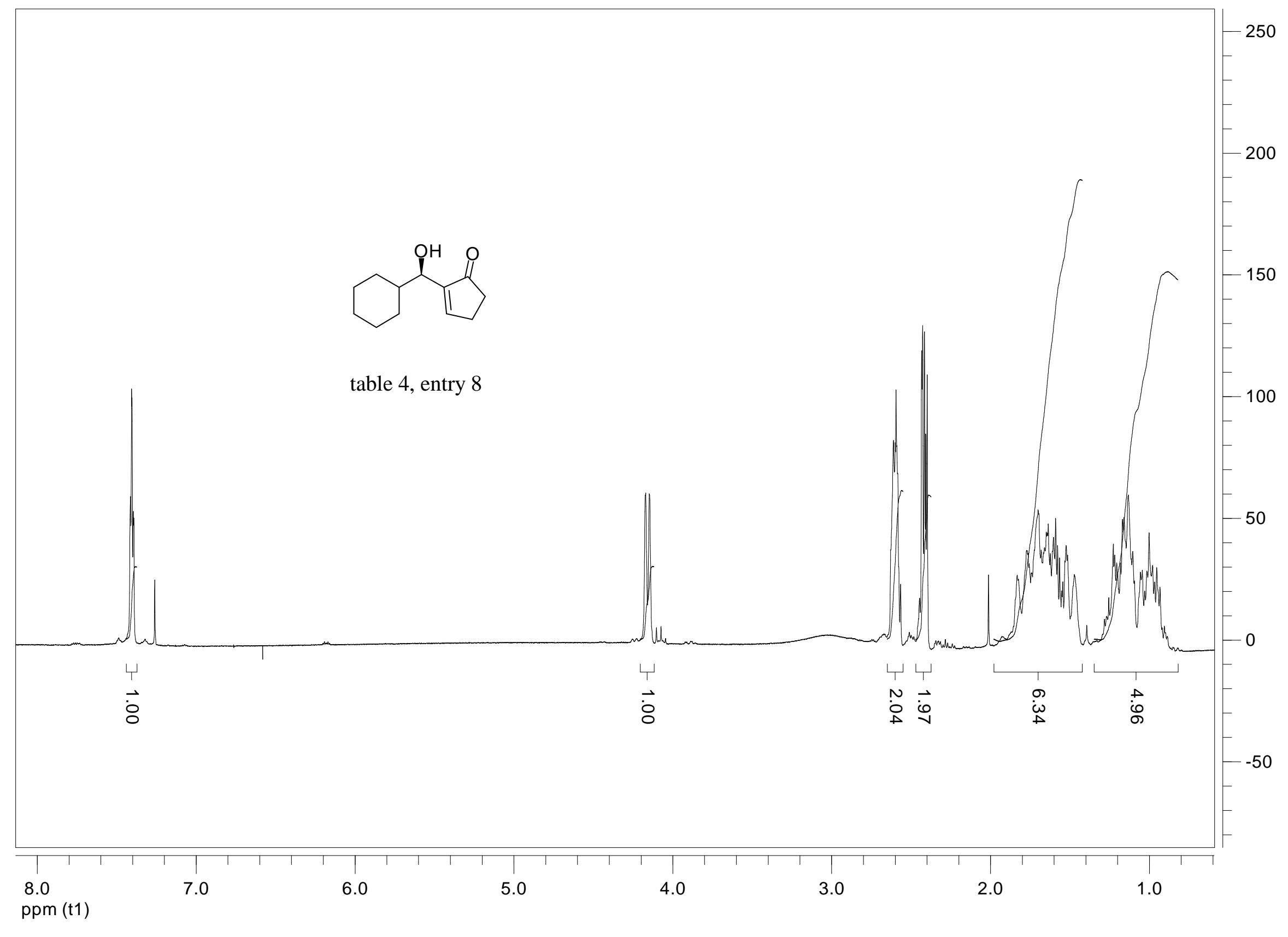




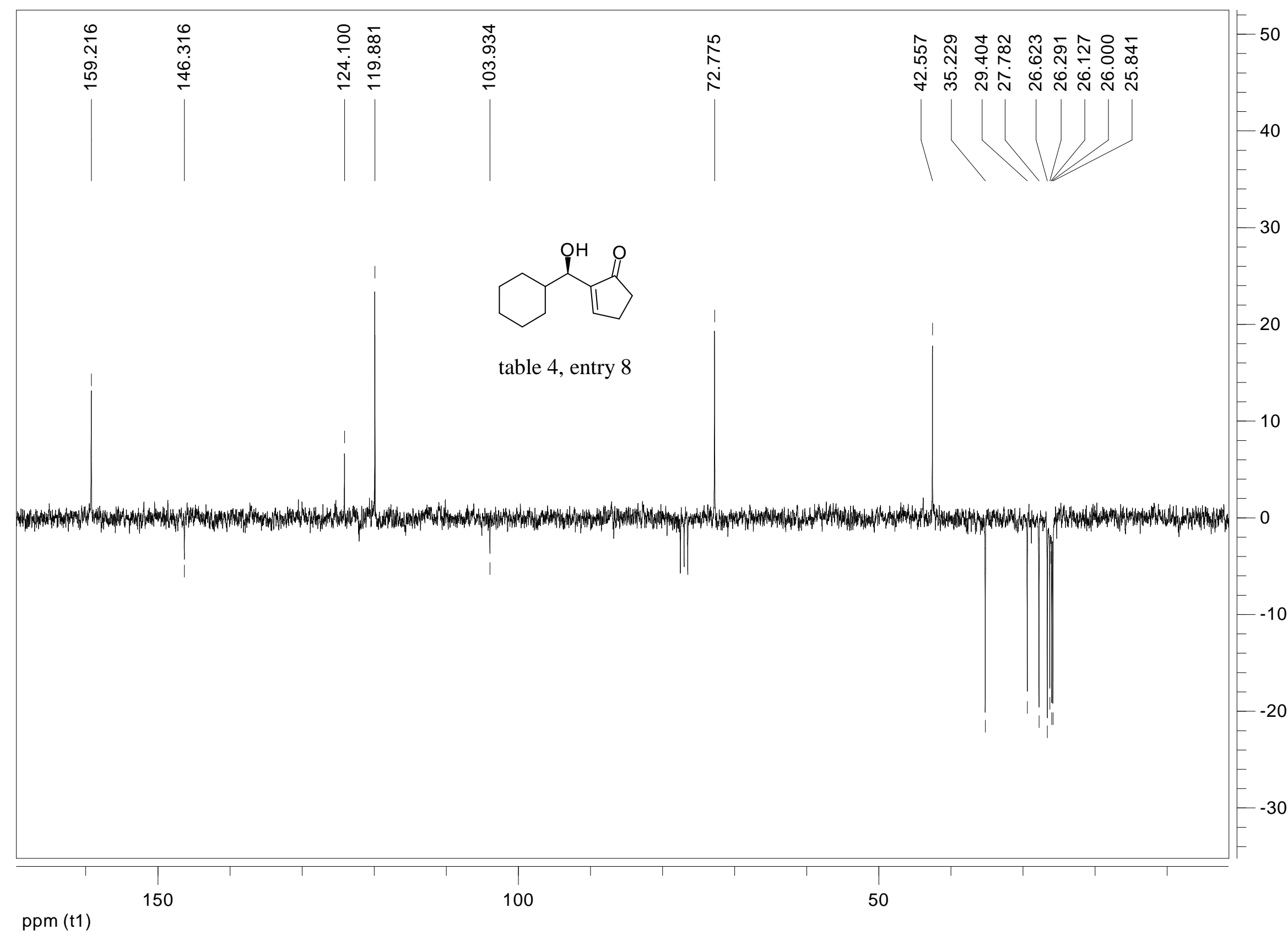




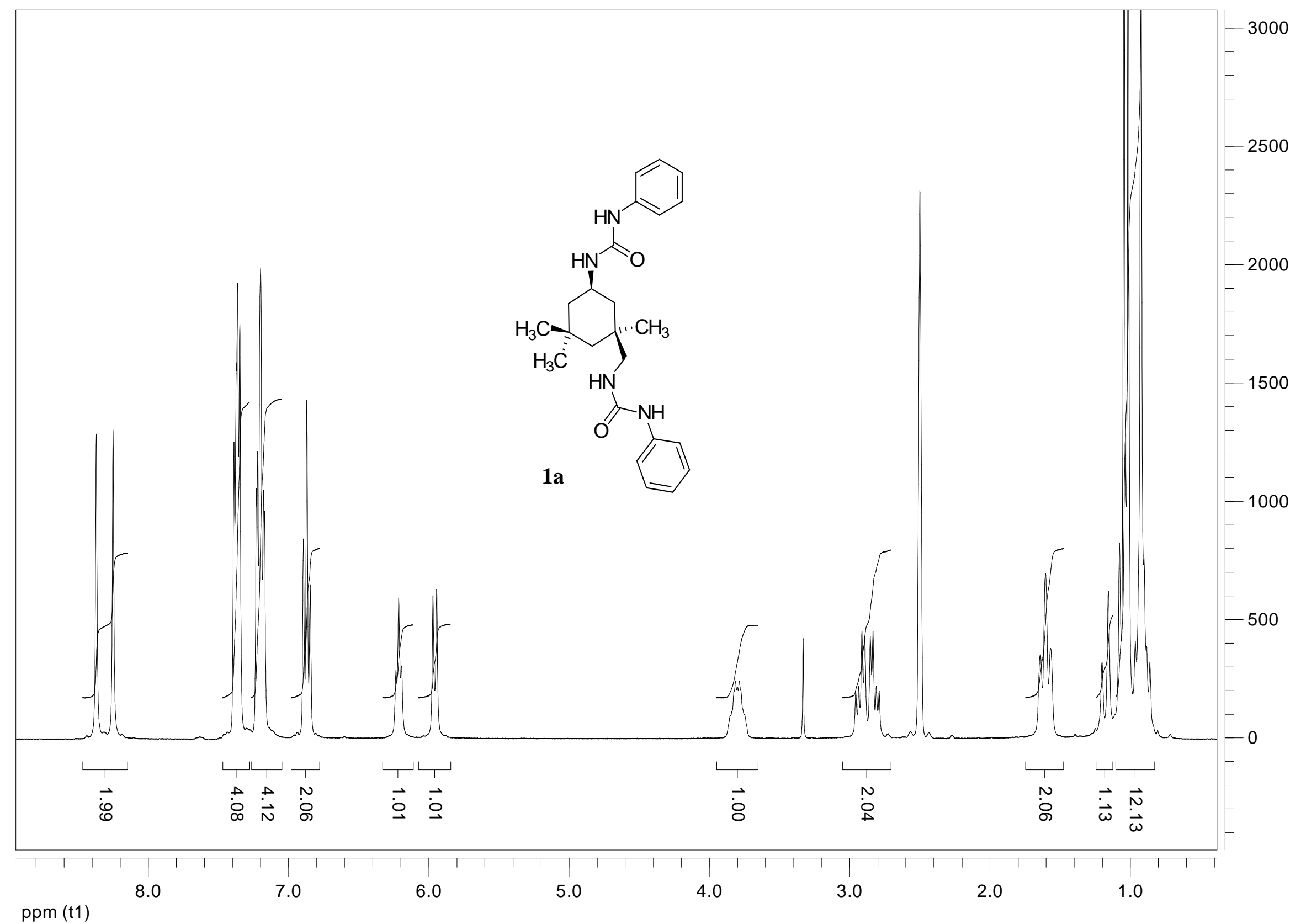




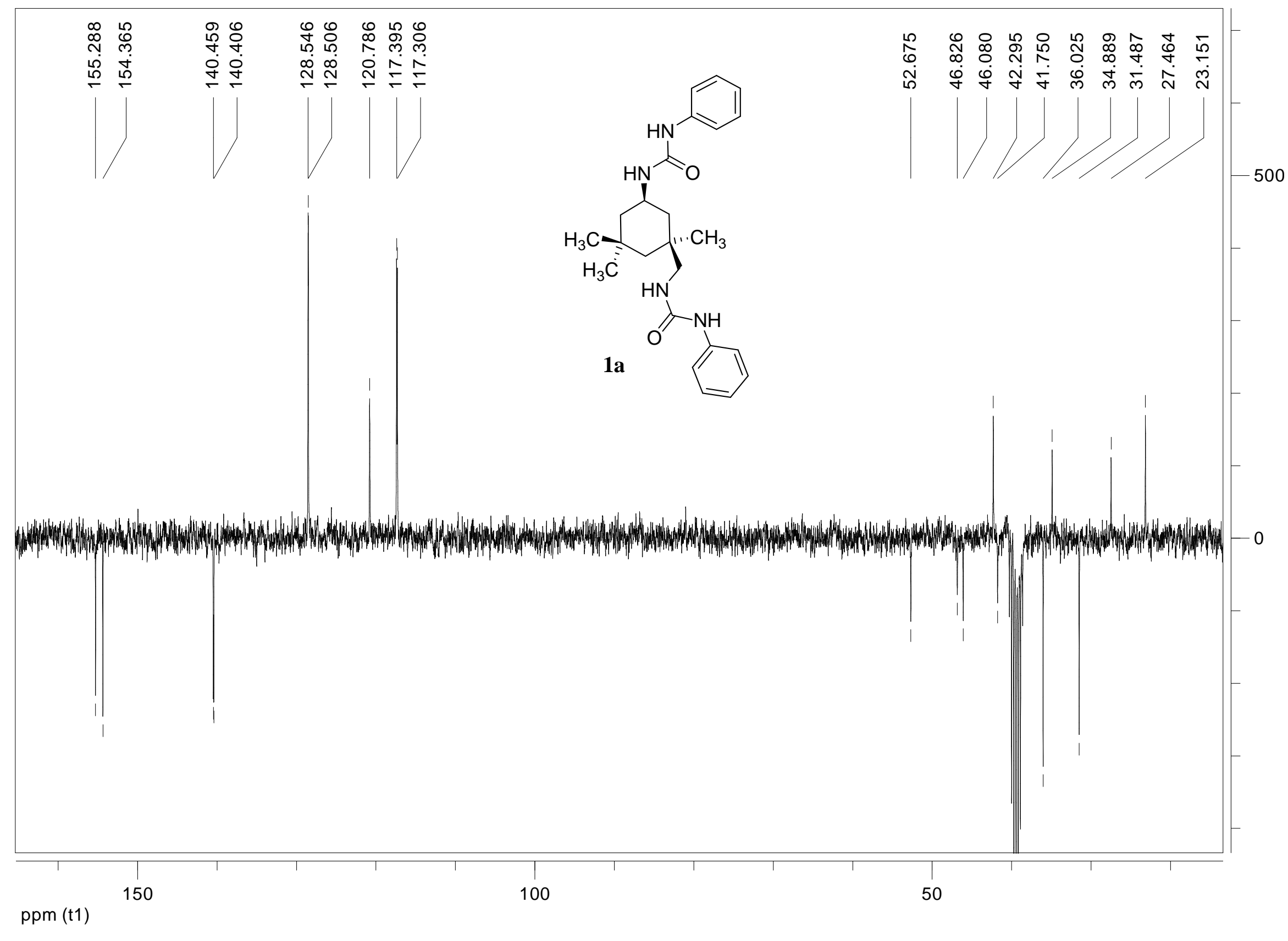




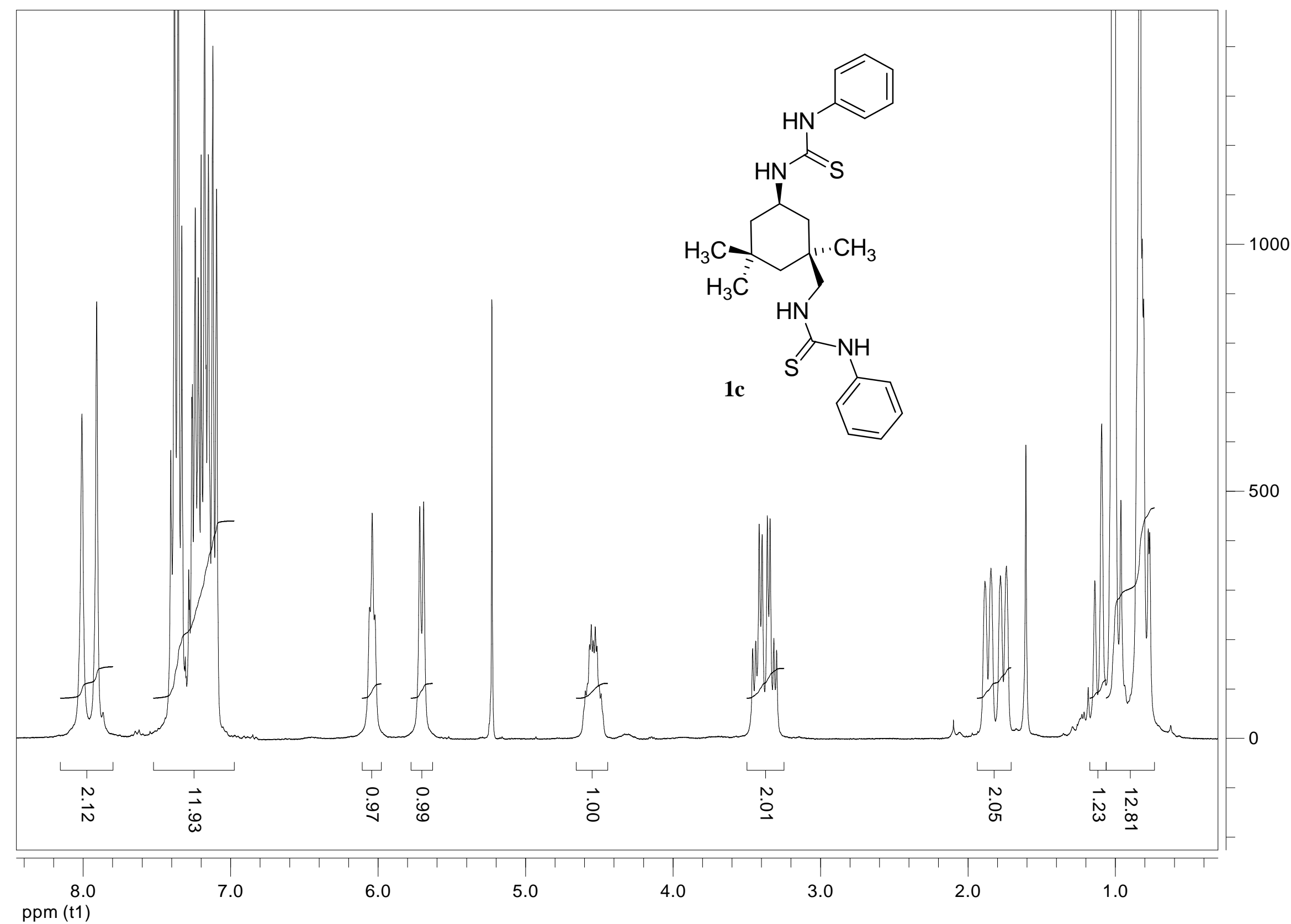




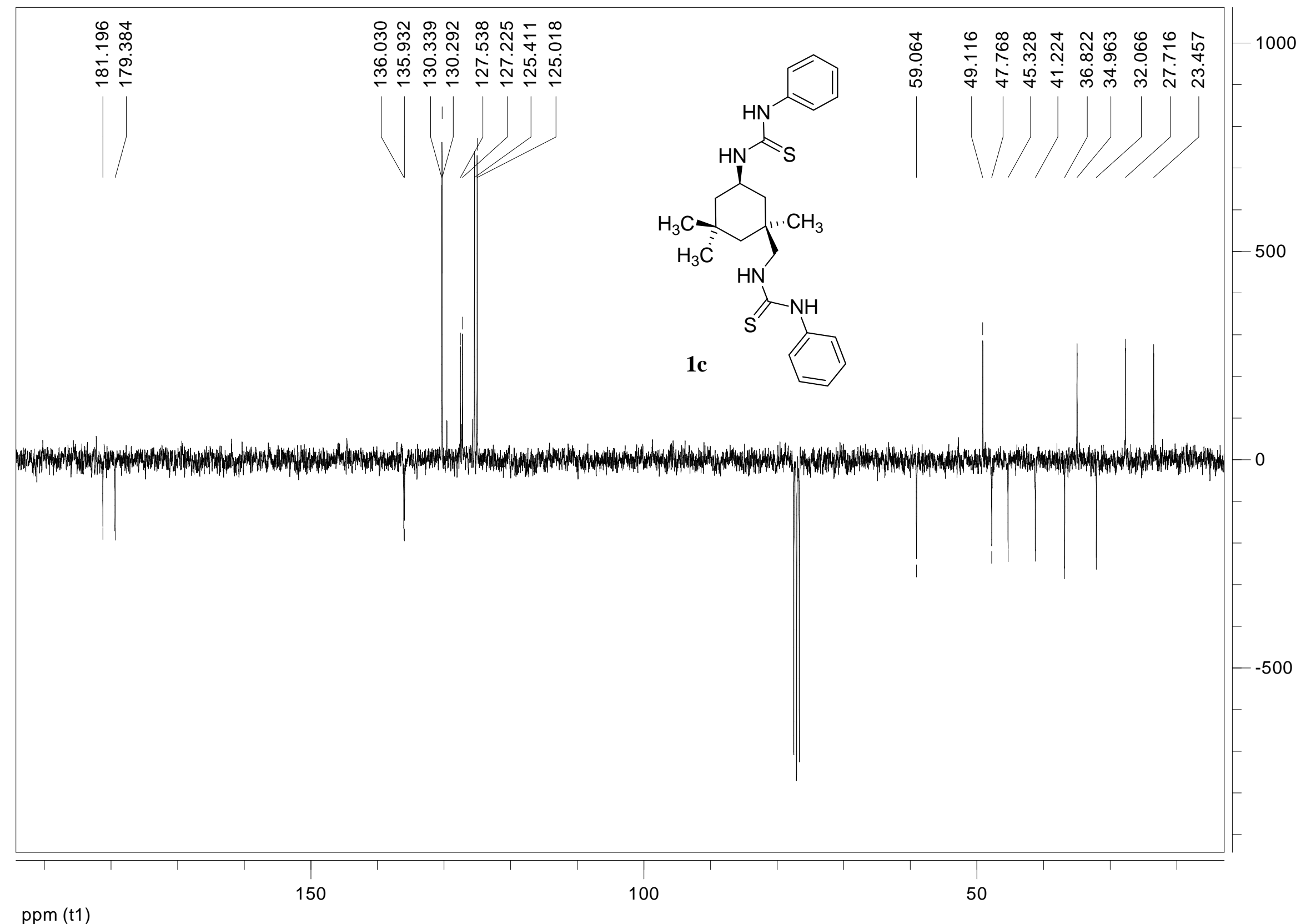




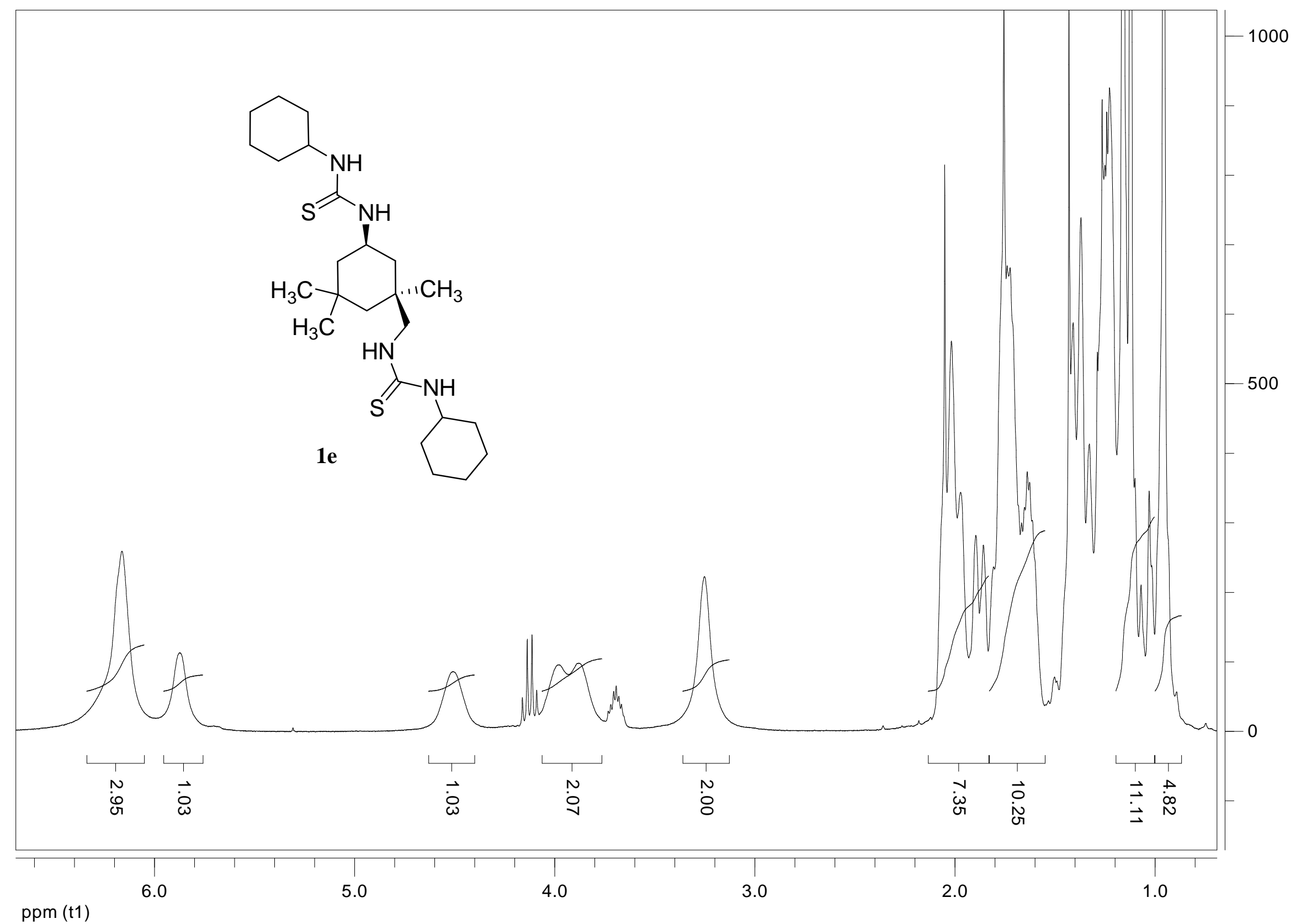




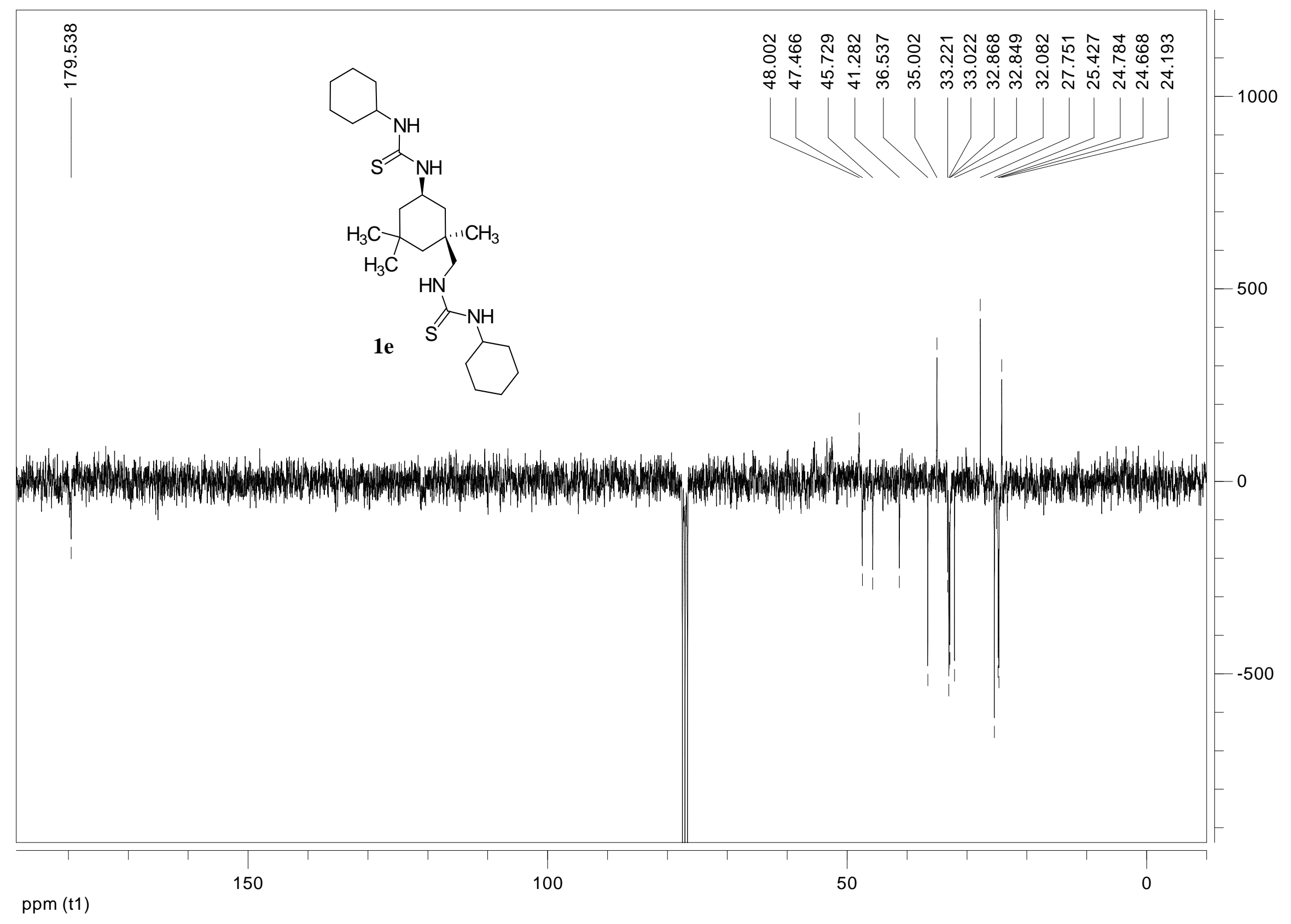




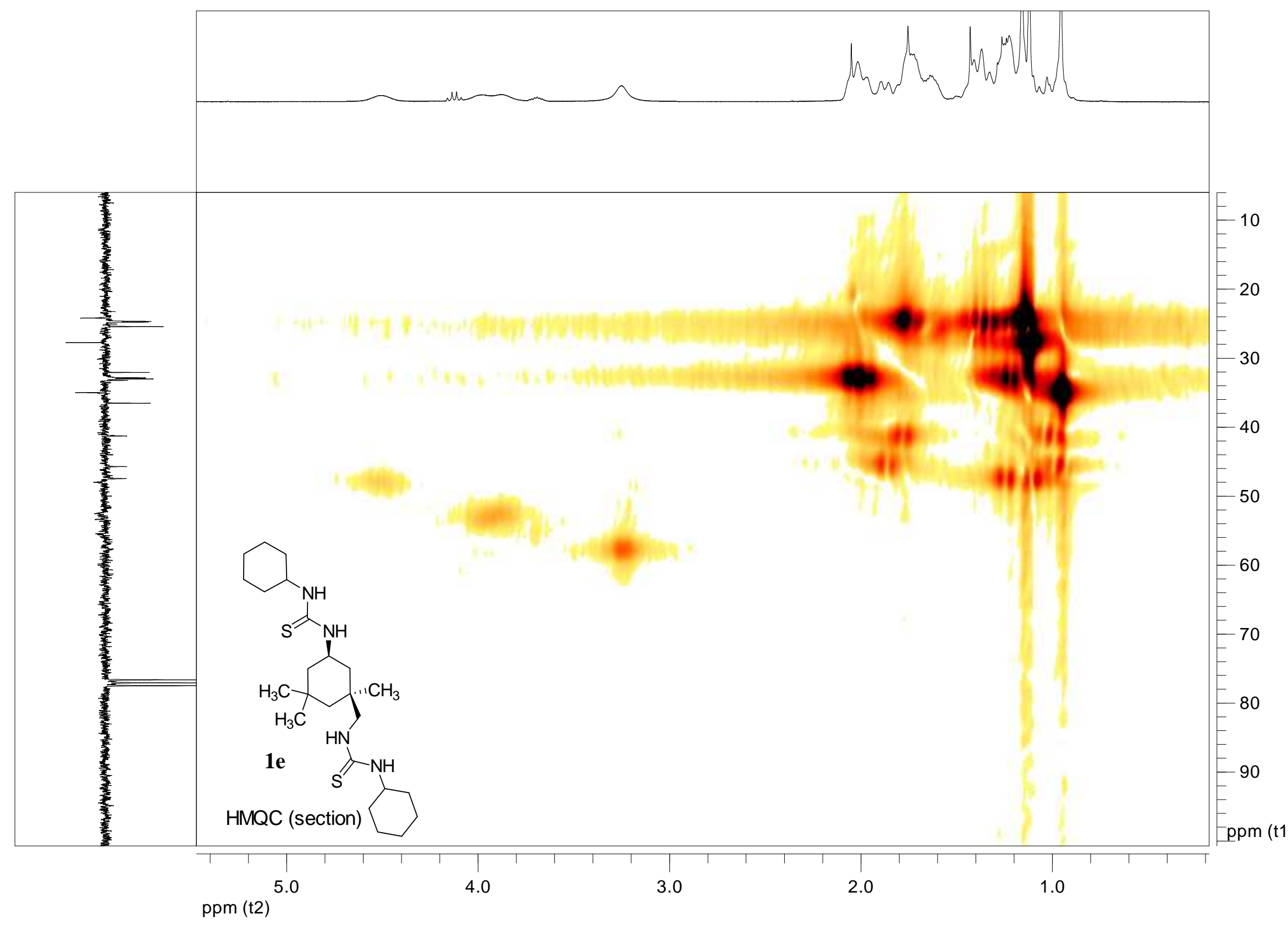




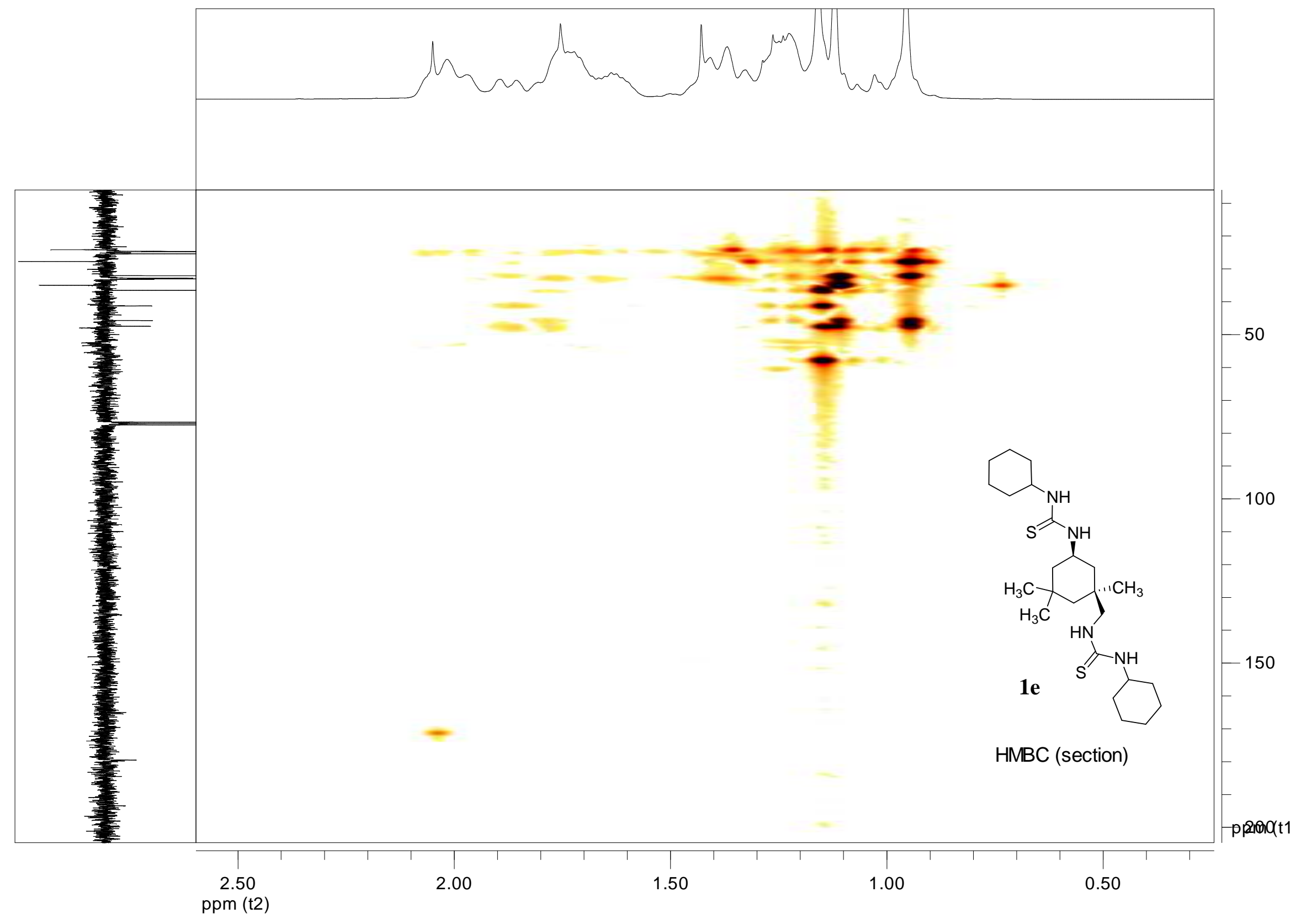




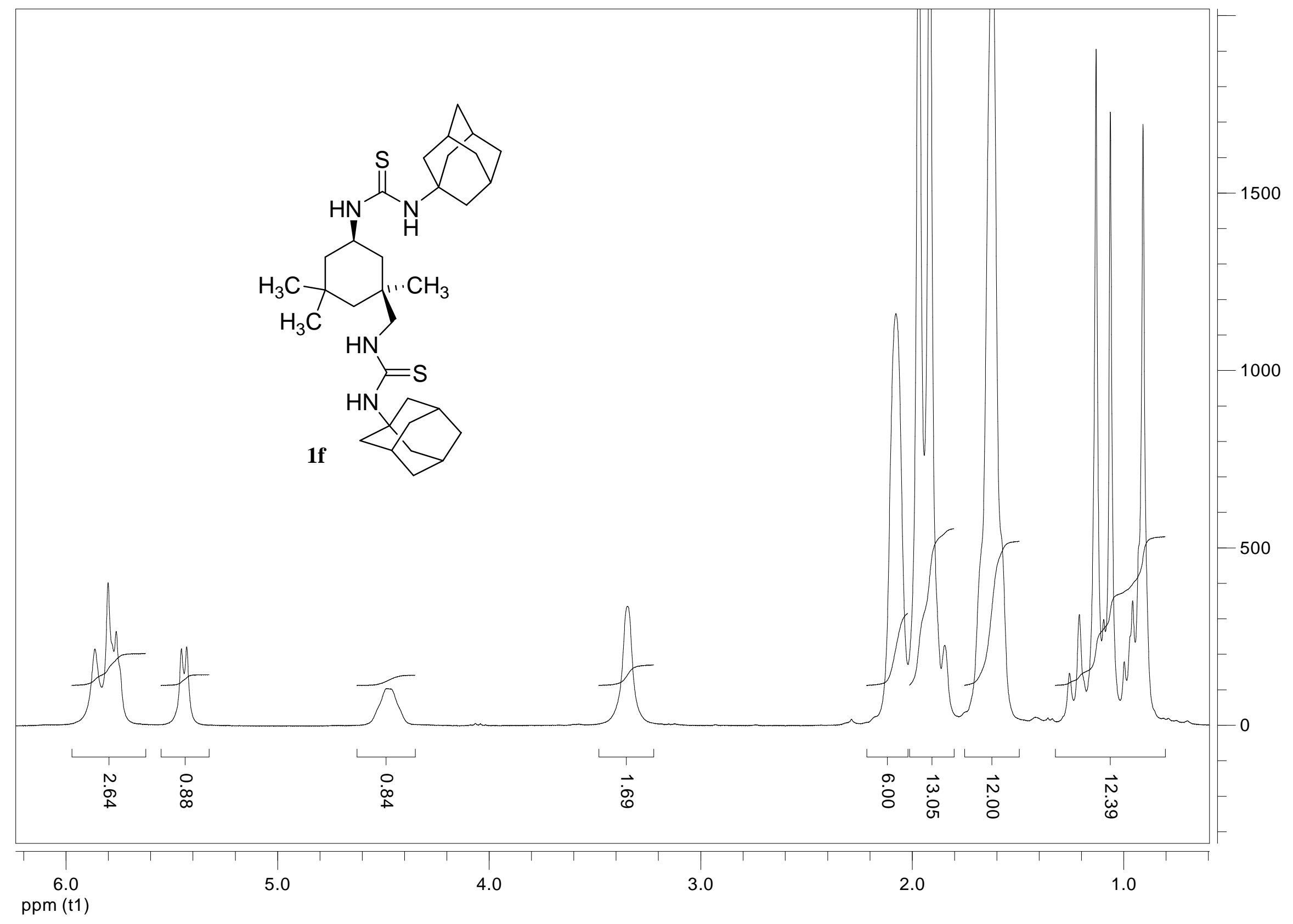




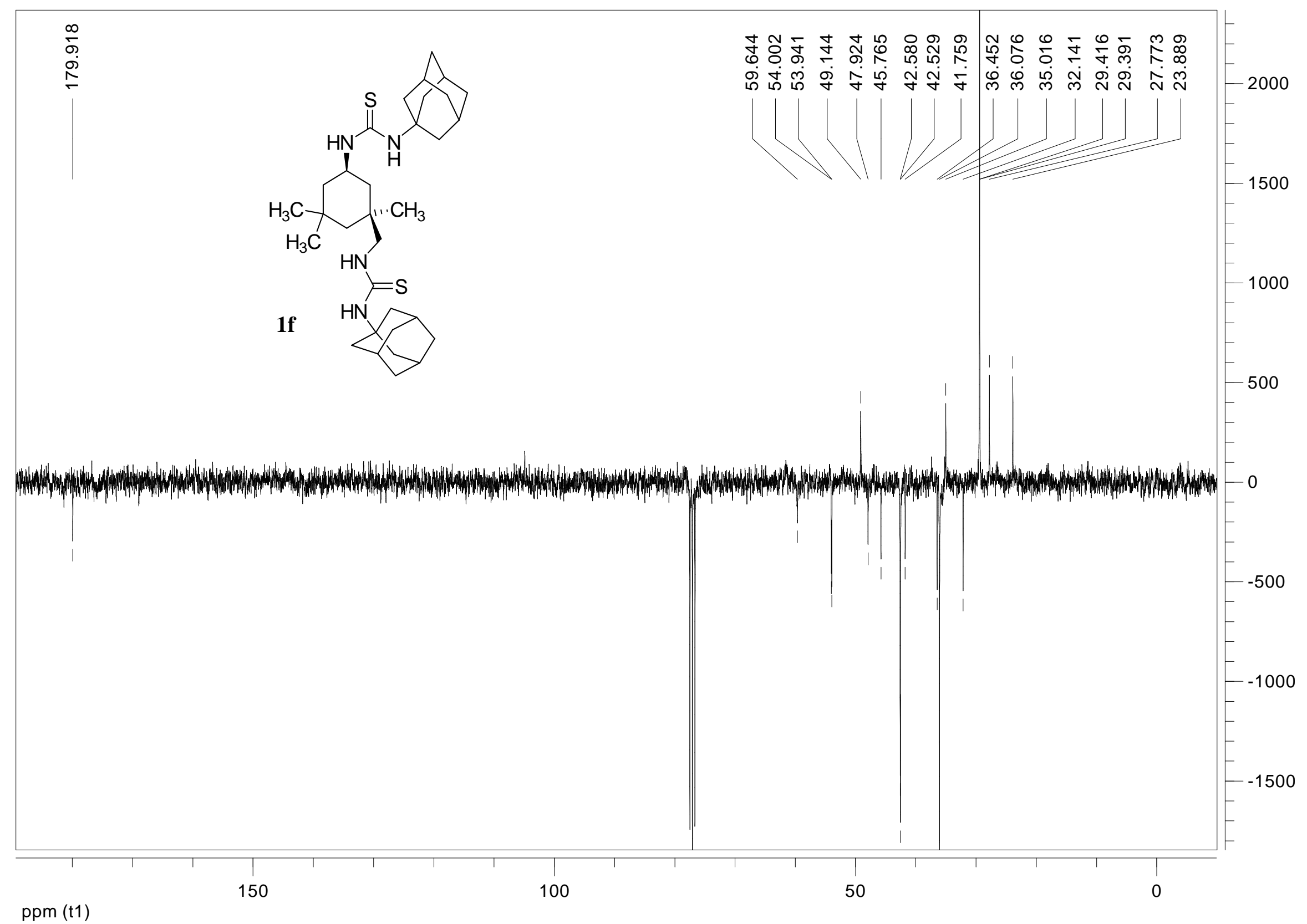




\section{References:}

1. Armarego, W. L. F.; Perrin, D. D. Purification of Laboratory Chemicals; Butterworth Heinemann: Oxford, 1996.

2. Aggarwal, V. K.; Mereu, A. Chem. Commun. 1999, 2311.

3. Sohtome, Y.; Tanatani, A., Hashimoto, Y., Nagasawa, K. Tetrahedron Lett. 2004, 45, 5589.

4. Luo, S.; Wang, P. G.; Cheng, J.-P. J. Org. Chem. 2004, 69, 555.

5. (a) McDougal, N. T.; Trevellini, W. L.; Rodgen, S. A.; Kliman, L. T.; Schaus, S. E. Adv. Synth. Catal. 2004, 346, 1231. (b) McDougal, N. R.; Schaus, S. E. J. Am. Chem. Soc. 2003, 125, 12094.

6. Lee, K. Y.; Gowrisankar, S.; Kim, J. N. Tetrahedron Lett. 2004, 45, 5485.

7. Marson, C. M.; Benzies, D. W. M.; Hobson, A. D. Tetrahedron 1991, 47, 5491.

8. Matsui, K.; Takizawa, S.; Sasai, H. Tetrahedron Lett. 2005, 46, 1943.

9. Aggarwal, V. K.; Mereu, A.; Tarver, G. J.; McCague, R. J. Org. Chem. 1998, 63, 7183. 